\title{
WIENER INDEX OF TREES OF GIVEN ORDER AND DIAMETER AT MOST 6
}

\author{
SIMON MUKWEMBI and TOMÁŠ VETRÍK ${ }^{凶}$
}

(Received 8 May 2013; accepted 25 July 2013; first published online 19 September 2013)

\begin{abstract}
The long-standing open problem of finding an upper bound for the Wiener index of a graph in terms of its order and diameter is addressed. Sharp upper bounds are presented for the Wiener index, and the related degree distance and Gutman index, for trees of order $n$ and diameter at most 6 .
\end{abstract}

2010 Mathematics subject classification: primary 05C12; secondary 05C05, $05 \mathrm{C} 07$.

Keywords and phrases: Wiener index, degree distance, Gutman index, tree, diameter.

\section{Introduction}

Let $G$ be a graph with vertex set $V(G)$ and order $n$. We denote the distance between two vertices $u, v$ in $G$ by $d_{G}(u, v)$ (or simply $d(u, v)$ ); the diameter of $G$ will be denoted by $d(G)$ (or $d$ ), the eccentricity of a vertex $v$ will be denoted by ec( $(v)$ and the degree of $v$ will be denoted by $\operatorname{deg}(v)$. Let $N_{i}^{G}(v)$ (or simply $N_{i}(v)$ ) be the set of vertices at distance $i$ from $v$ in $G$. Let $u, v$ be two adjacent (nonadjacent) vertices of a graph $G$. Then $G^{\prime}=G-u v\left(G^{\prime}=G+u v\right)$ is obtained by removing the edge $u v$ from $G$ (by adding the edge $u v$ to $G)$.

The Wiener index is the oldest topological index. It has been investigated in the mathematical, chemical and computer science literature since the 1940s. The Wiener index $W(G)$ of a connected graph $G$ is defined as the sum of the distances between all unordered pairs of vertices. The minimum value of the Wiener index of a graph (of a tree) of given order is attained by the complete graph (by the star), and the maximum value is attained by the path.

The degree distance, a variant of the Wiener index, is defined as

$$
D^{\prime}(G)=\sum_{\{u, v\} \subseteq V(G)}(\operatorname{deg}(u)+\operatorname{deg}(v)) d(u, v),
$$

Financial support by the National Research Foundation and the University of KwaZulu-Natal is gratefully acknowledged.

(c) 2013 Australian Mathematical Publishing Association Inc. 0004-9727/2013 \$16.00 
and the Gutman index is defined as

$$
\operatorname{Gut}(G)=\sum_{\{u, v\} \subseteq V(G)} \operatorname{deg}(u) \operatorname{deg}(v) d(u, v) .
$$

The smallest value of the degree distance and Gutman index of graphs of order $n$ is attained by stars (see [1, 11]). Turning to upper bounds on the degree distance, in 1999 Tomescu [11] conjectured the asymptotic upper bound $D^{\prime}(G) \leq(1 / 27) n^{4}+$ $O\left(n^{3}\right)$. Nine years later, Bucicovschi and Cioabă [2] commented that Tomescu's conjecture 'seems difficult at present time'. In the following year Dankelmann et al. [3] considered this problem and though they came close to proving the conjecture, their proof was inadequate to meet the $O\left(n^{3}\right)$ error term. Recently, Morgan et al., in a submitted paper ('On a conjecture by Tomescu'), salvaged enough from the proof given in [3] and solved Tomescu's conjecture completely. There one can also find upper bounds on the degree distance of graphs of given order and diameter. Upper bounds on the Gutman index of a graph of given order and diameter were studied in [4, 9]. In [9] it was proved, that $\operatorname{Gut}(G) \leq(1 / 16) d(n-d)^{4}+O\left(n^{4}\right)$ and consequently $\operatorname{Gut}(G) \leq\left(2^{4} / 5^{5}\right) n^{5}+O\left(n^{4}\right)$.

In this paper we study the indices mentioned above for trees of given order and diameter. Since Klein et al. [7] showed that for every tree $T$ of order $n$,

$$
D^{\prime}(T)=4 W(T)-n(n-1),
$$

and in [6] Gutman proved that

$$
\operatorname{Gut}(T)=4 W(T)-(2 n-1)(n-1)
$$

any result on $W(T)$ yields a similar result on $D^{\prime}(T)$ and $\operatorname{Gut}(T)$. It is not difficult to show that the extremal tree, which has the minimum Wiener index among trees of order $n$ and diameter $d$, is the path of length $d$ (containing $d+1$ vertices) with the central vertex joined to the other $n-d-1$ vertices; see [12].

The problem of finding an upper bound on the Wiener index of a tree (or graph) in terms of order and diameter is quite challenging; it was addressed by Plesník [10] in 1975, and restated by DeLaViña and Waller [5], but still remains unresolved to this date. In this paper, we give a starting point to solving this long-standing problem. We present upper bounds on the Wiener index of trees of order $n$ and diameter at most 6 , and we show that our bounds are best possible. As a corollary we obtain upper bounds on the degree distance and Gutman index of trees of given order and diameter at most 6. Let us mention that there are indices which were introduced much later than the Wiener index, however upper bounds on these indices for trees of given order and diameter are known. For example, a sharp upper bound on the eccentric connectivity index of trees of given order and diameter was given in [8]. To find a sharp upper bound on the Wiener index for trees of given order and large diameter seems to be a very complicated problem. 


\section{Preliminary results}

First we give a few results which will be used in proofs of our main theorems. Note that

$$
W(T)=\sum_{\{u, v\} \subseteq V(T)} d(u, v)=\frac{1}{2} \sum_{u \in V(T)} \sum_{v \in V(T)} d(u, v)=\frac{1}{2} \sum_{u \in V(T)} \sum_{i=1}^{d} i\left|N_{i}^{T}(u)\right| .
$$

Lemma 2.1. Let $T$ be a tree of diameter $2 r(r \geq 2)$ with the central vertex $v$, and let $\operatorname{deg}(u)=2$ for every vertex $u \in N_{i}(v)$ where $i=1,2, \ldots, r-2$. If $T$ has the maximum Wiener index among trees of given order and diameter $2 r$, then the degrees of any two vertices in $N_{r-1}(v)$ differ by at most one.

Proof. Let $u_{1}, u_{2}$ be any two vertices in $N_{r-1}^{T}(v)$, and let $n_{i}$ be the number of leaves adjacent to $u_{i}$ in $T, i=1,2$. We prove the result by contradiction. Suppose that $n_{1} \geq n_{2}+2$. We show that $T$ does not have the maximum Wiener index among trees of given order and diameter $2 r$. Let $w$ be any leaf adjacent to $u_{1}$ in $T$, and let $T^{\prime}=$ $T-u_{1} w+u_{2} w$. We have $V\left(T^{\prime}\right)=V(T), d\left(T^{\prime}\right)=d(T)=2 r, d_{T}\left(w_{1}, w_{2}\right)=d_{T^{\prime}}\left(w_{1}, w_{2}\right)$ for any two vertices $w_{1}, w_{2}$ different from $w$, and $\left|N_{i}^{T}(w)\right| \neq\left|N_{i}^{T^{\prime}}(w)\right|$ only if $i=2$ or $2 r$. Since $\left|N_{2}^{T}(w)\right|=n_{1},\left|N_{2}^{T^{\prime}}(w)\right|=n_{2}+1$ and $\left|N_{2 r}^{T^{\prime}}(w)\right|-\left|N_{2 r}^{T}(w)\right|=\left(n_{1}-1\right)-n_{2}$,

$$
\begin{aligned}
W\left(T^{\prime}\right)-W(T) & =2 r\left(\left|N_{2 r}^{T^{\prime}}(w)\right|-\left|N_{2 r}^{T}(w)\right|\right)+2\left(\left|N_{2}^{T^{\prime}}(w)\right|-\left|N_{2}^{T}(w)\right|\right) \\
& =2(r-1)\left(n_{1}-n_{2}-1\right)>0,
\end{aligned}
$$

which is a contradiction.

Corollary 2.2. Let $T_{1}$ be a join of a tree $T$ (which is defined in the previous lemma) and any tree $T_{2}$, where $T_{1}$ is constructed in such a way that we unify the central vertex of $T$ with any vertex of $T_{2}$. If $T_{1}$ has the maximum Wiener index among trees of given order and diameter, then the degrees of any two vertices in $N_{r-1}(v)$ which are in $T$ differ by at most one.

Lemma 2.3. Let $T$ be a tree of diameter $2 r(r \geq 2)$ with the central vertex $v$, and let $\operatorname{deg}(u)=2$ for every vertex $u \in N_{i}(v)$ where $i=1,2, \ldots, r-2$. Let $|N(v)|=k$ and $\left|N_{r}(v)\right|=n_{k}$. If $T$ has the maximum Wiener index among trees of given order and diameter $2 r$, then

$$
\sum_{\{y, x\} \subseteq N_{r}(v)} d(y, x) \leq n_{k}\left(r n_{k}+(1-r) \frac{n_{k}}{k}-1\right),
$$

and we have the equality only if the degrees of all vertices in $N_{r-1}(v)$ are equal.

Proof. Let $T$ be a tree with $\operatorname{deg}(u)=2$ for every vertex $u \in N_{i}(v)$ where $i=$ $1,2, \ldots, r-2$ and let $|N(v)|=k$. Then $\left|N_{i}(v)\right|=k$ for any $i=2,3, \ldots, r-1$. Let $N_{r-1}(v)=\left\{v_{1}, v_{2}, \ldots, v_{k}\right\}$. By Lemma 2.1, if $T$ has the maximum Wiener index, then $v_{j}$ $(j=1,2, \ldots, k)$ has either $s-1$ or $s$ neighbours in $N_{r}(v)$ for some $s \geq 1$. Without loss 
of generality, we can assume that the number of vertices in $N_{r}(v)$ which are adjacent to $v_{i}(i=1,2, \ldots, p, 1 \leq p \leq k)$ is $s-1$, and the number of vertices in $N_{r}(v)$ which are adjacent to $v_{j}(j=p+1, p+2, \ldots, k)$ is $s$. We have $n_{k}=p(s-1)+(k-p) s=k s-p$. Then any two vertices in $N_{r}(v)$ are of distance 2 if they have a common neighbour in $N_{r-1}(v)$, otherwise they are of distance $2 r$. Hence, for $w, w^{\prime} \in N_{r}(v)$,

$$
\begin{aligned}
& \sum_{x \in N_{r}(v)} d(w, x)=2(s-2)+2 r(k s-p-s+1) \quad \text { if } w \in N\left(v_{i}\right), i=1,2, \ldots, p, \\
& \sum_{x \in N_{r}(v)} d\left(w^{\prime}, x\right)=2(s-1)+2 r(k s-p-s) \quad \text { if } w^{\prime} \in N\left(v_{j}\right), j=p+1, p+2, \ldots, k,
\end{aligned}
$$

which yields

$$
\begin{aligned}
2 \sum_{\{y, x\} \subseteq N_{r}(v)} d(y, x) & =\sum_{y \in N_{r}(v)} \sum_{x \in N_{r}(v)} d(y, x) \\
& =p(s-1) \sum_{x \in N_{r}(v)} d(w, x)+(k-p) s \sum_{x \in N_{r}(v)} d\left(w^{\prime}, x\right) \\
& =(k s-p)(2 r(k s-p)+2(1-r) s-2)+2 p(s-1)(r-1) .
\end{aligned}
$$

Since $p / k \leq 1$, we have $s-1 \leq s-p / k$, and consequently

$$
2 p(s-1)(r-1) \leq 2 p\left(s-\frac{p}{k}\right)(r-1)=\frac{2 p}{k}(k s-p)(r-1) .
$$

Hence

$$
\begin{aligned}
\sum_{\{y, x\} \subseteq N_{r}(v)} d(y, x) & \leq \frac{k s-p}{2}\left(2 r(k s-p)+2(1-r) s+\frac{2 p}{k}(r-1)-2\right) \\
& =n_{k}\left(r n_{k}+(1-r) \frac{n_{k}}{k}-1\right) .
\end{aligned}
$$

Clearly we have equality above only if $p / k=1$, which means that every vertex in $N_{r-1}(v)$ is adjacent to $s-1$ vertices in $N_{r}(v)$.

Corollary 2.4. Let $T_{1}$ be a join of a tree $T$ (defined as in Lemma 2.3) and a new tree $T_{2}$, where $T_{1}$ is constructed in such a way that we unify the central vertex of $T$ with any vertex of $T_{2}$. Then the distances between vertices in $T$ do not change, and if $T_{1}$ has the maximum Wiener index among trees of given order and diameter, then

$$
\sum_{\{y, x\} \subseteq N_{r}^{T}(v)} d(y, x) \leq n_{k}\left(r n_{k}+(1-r) \frac{n_{k}}{k}-1\right),
$$

and we have equality only if the degrees of all vertices in $N_{r-1}^{T}(v)$ are equal.

LEMMA 2.5. Let $u_{1}, u_{2}, \ldots, u_{k}$ be any set of vertices of a tree $T$ which have a common neighbour, and let all the other neighbours of $u_{i}$ be leaves, $i=1,2, \ldots, k$. If $T$ has the maximum Wiener index among trees of order $n$ and diameter $d \geq 5$, then: 
(i) if $k \geq 2$ and $\operatorname{ec}\left(u_{i}\right)<d$, then $\left|N\left(u_{i}\right)\right|+\left|N\left(u_{j}\right)\right|>\sqrt{2 n}-1$ for any $i, j \in$ $\{1,2, \ldots, k\}, i \neq j$;

(ii) $\left|N\left(u_{i}\right)\right|<\sqrt{2 n}+1$ for any $i \in\{1,2, \ldots, k\}$.

Proof. Let $u$ be a neighbour of all $u_{i}, i=1,2, \ldots, k$, and let $U_{i}=N\left(u_{i}\right) \backslash\{u\}$. We prove by contradiction that $\left|U_{i}\right|<\sqrt{2 n}$ and if $k \geq 2$ and ec $\left(u_{i}\right)<d$, then $\left|U_{i}\right|+\left|U_{j}\right|>\sqrt{2 n}-3$ for any $i, j \in\{1,2, \ldots, k\}, i \neq j$.

(i) Suppose that there are 2 vertices $u_{i}, u_{j}$ such that $\left|U_{i}\right|+\left|U_{j}\right| \leq \sqrt{2 n}-3$. Let

$$
T^{\prime}=T-\bigcup_{w \in U_{j}} u_{j} w-u u_{j}+\bigcup_{w \in U_{j}} u_{i} w+u_{i} u_{j}
$$

Note that if we do not assume that $\operatorname{ec}\left(u_{i}\right)<d(T)$, then $u_{i}$ can be the end vertex of a diametral path in $T$, which implies $d(T)<d\left(T^{\prime}\right)$. We also know that (since $d(T) \geq 5$ ) there is a vertex, say $y$, such that $d_{T}(v, y)=d_{T^{\prime}}(v, y) \geq 3$, and hence $d\left(T^{\prime}\right)$ cannot be less than 5. It follows that $d(T)=d\left(T^{\prime}\right)$ and $d_{T}\left(w_{1}, w_{2}\right)=d_{T^{\prime}}\left(w_{1}, w_{2}\right)$ for any two vertices $w_{1}, w_{2}$ except for the cases when $w_{1} \in U_{i} \cup\left\{u_{i}\right\}$ and $w_{2} \in U_{j}$, or when $w_{1}=u_{j}$. We have

$$
\begin{gathered}
d_{T^{\prime}}\left(w_{1}, w_{2}\right)=d_{T}\left(w_{1}, w_{2}\right)-2 \quad \text { if } w_{1} \in U_{i} \cup\left\{u_{i}\right\}, w_{2} \in U_{j}, \\
d_{T^{\prime}}\left(u_{j}, w\right)=d_{T}\left(u_{j}, w\right)-1 \quad \text { if } w \in U_{i} \cup\left\{u_{i}\right\}, \\
d_{T^{\prime}}\left(u_{j}, w\right)=d_{T}\left(u_{j}, w\right)+1 \text { if } w \in V(T) \backslash\left(U_{i} \cup\left\{u_{i}, u_{j}\right\}\right) .
\end{gathered}
$$

Hence

$$
\begin{aligned}
W\left(T^{\prime}\right)-W(T)= & \sum_{w_{1} \in U_{i} \cup\left\{u_{i}\right\}} \sum_{w_{2} \in U_{j}}\left(d_{T^{\prime}}\left(w_{1}, w_{2}\right)-d_{T}\left(w_{1}, w_{2}\right)\right) \\
& \quad+\sum_{w \in V(T)}\left(d_{T^{\prime}}\left(u_{j}, w\right)-d_{T}\left(u_{j}, w\right)\right) \\
= & -2\left(\left|U_{i}\right|+1\right)\left|U_{j}\right|-\left(\left|U_{i}\right|+1\right)+\left(n-\left|U_{i}\right|-2\right) \\
= & n-2\left|U_{i}\right|\left|U_{j}\right|-2\left|U_{i}\right|-2\left|U_{j}\right|-3 .
\end{aligned}
$$

Since $\left|U_{i}\right|\left|U_{j}\right| \leq\left(\left(\left|U_{i}\right|+\left|U_{j}\right|\right) / 2\right)^{2}$,

$$
W\left(T^{\prime}\right)-W(T) \geq n-2\left(\frac{\sqrt{2 n}-3}{2}\right)^{2}-2(\sqrt{2 n}-3)-3=\sqrt{2 n}-\frac{3}{2}>0 .
$$

Hence $T$ is not a graph with the maximum Wiener index.

(ii) Suppose that $\left|U_{i}\right| \geq \sqrt{2 n}$ for some $i \in\{1,2, \ldots, k\}$. Let $x \in U_{i}$, and let $X$ and $Y$ be two disjoint subsets of $U_{i}$ such that $|X|$ and $|Y|$ differ by at most 1 , and $U_{i}=X \cup Y \cup\{x\}$. Then $|X|,|Y| \geq \sqrt{n / 2}-1$. Let

$$
T^{\prime}=T-\bigcup_{w \in X} u_{i} w-u_{i} x+u x+\bigcup_{w \in X} x w .
$$


Then $d_{T}\left(w_{1}, w_{2}\right) \neq d_{T^{\prime}}\left(w_{1}, w_{2}\right)$ only in the following cases:

$$
\begin{gathered}
d_{T^{\prime}}\left(w_{1}, w_{2}\right)=d_{T}\left(w_{1}, w_{2}\right)+2 \quad \text { if } w_{1} \in Y \cup\left\{u_{i}\right\}, w_{2} \in X, \\
d_{T^{\prime}}(x, w)=d_{T}(x, w)+1 \quad \text { if } w \in Y \cup\left\{u_{i}\right\}, \\
d_{T^{\prime}}(x, w)=d_{T}(x, w)-1 \quad \text { if } w \in V(T) \backslash\left(Y \cup\left\{u_{i}, x\right\}\right) .
\end{gathered}
$$

Hence

$$
\begin{aligned}
W(T)-W\left(T^{\prime}\right) & =\sum_{w_{1} \in Y \cup\left\{u_{i}\right\}} \sum_{w_{2} \in X}\left(d_{T}\left(w_{1}, w_{2}\right)-d_{T^{\prime}}\left(w_{1}, w_{2}\right)\right)+\sum_{w \in V(T)}\left(d_{T}(x, w)-d_{T^{\prime}}(x, w)\right) \\
& =-2(|Y|+1)|X|-(|Y|+1)+(n-|Y|-2) \\
& =n-2|X||Y|-2|X|-2|Y|-3 \\
& =n-2|X||Y|-2\left|U_{i}\right|-1 \\
& \leq n-2\left(\sqrt{\frac{n}{2}}-1\right)^{2}-2 \sqrt{2 n}-1=-3
\end{aligned}
$$

which is a contradiction.

\section{Main results}

We present results on the Wiener index of trees of given order and diameter at most 6. The only tree of order $n$ and diameter 2 is the star $S_{n}$ having $n-1$ leaves. Since any two leaves of the star are at distance 2 , and the distance between the central vertex and any leaf is 1 , the Wiener index of $S_{n}$ is $2\left(\begin{array}{c}n-1 \\ 2\end{array}\right)+(n-1)=n^{2}-2 n+1$. Then from (1.1) and (1.2) it follows that the degree distance of the star is $D^{\prime}\left(S_{n}\right)=$ $3 n^{2}-7 n+4$ and the Gutman index is $\operatorname{Gut}\left(S_{n}\right)=2 n^{2}-5 n+3$.

Now we bound the Wiener index for diameter $d$ where $3 \leq d \leq 6$.

Theorem 3.1. Let $T$ be a tree of order $n$ and diameter 3. Then the Wiener index of $T$ is

$$
W(T) \leq \frac{5 n^{2}}{4}-3 n+3
$$

and this bound is best possible.

Proof. Let $T$ be any tree of order $n$ and diameter 3. We denote the central vertices of $T$ by $v$ and $u$. The set of leaves adjacent to $v$ (to $u$ ) will be denoted by $K$ (by $L$ ). Let $|K|=k$. Then $|L|=n-k-2$. It can be checked that

$$
\begin{gathered}
\sum_{\{y, x\} \subseteq K} d(y, x)=2\left(\begin{array}{l}
k \\
2
\end{array}\right), \quad \sum_{\{y, x\} \subseteq L} d(y, x)=2\left(\begin{array}{c}
n-k-2 \\
2
\end{array}\right), \quad \sum_{y \in K} \sum_{x \in L} d(y, x)=3 k(n-k-2), \\
\sum_{x \in V(T)} d(v, x)=(k+1)+2(n-k-2) \quad \text { and } \quad \sum_{x \in V(T)} d(u, x)=(n-k-1)+2 k,
\end{gathered}
$$


which yield

$$
\begin{aligned}
W(T) & =\sum_{\{y, x\} \subseteq K} d(y, x)+\sum_{\{y, x\} \subseteq L} d(y, x)+\sum_{y \in K} \sum_{x \in L} d(y, x)+\sum_{x \in V(T)} d(v, x)+\sum_{x \in V(T)} d(u, x) \\
& =n^{2}-2 n+k n-k^{2}-2 k+2=f(k) .
\end{aligned}
$$

Then from the derivative $f^{\prime}(k)=0$ we obtain $k=n / 2-1$, which yields the maximum of $f(k)$. Hence

$$
W(T) \leq f\left(\frac{n}{2}-1\right)=\frac{5 n^{2}}{4}-3 n+3 .
$$

This value is attained by the Wiener index of a tree which has both central vertices of degree $n / 2$. Therefore our bound is best possible.

Theorem 3.2. Let $T$ be a tree of order $n$ and diameter 4 . Then

$$
W(T) \leq 2 n^{2}-2 n \sqrt{n-1}-3 n+2 \sqrt{n-1}+1
$$

and the bound is best possible.

Proof. Let $T$ be a tree with the maximal Wiener index among all trees of order $n$ and diameter 4. We denote the central vertex of $T$ by $v$. Let $|N(v)|=k$ and $\left|N_{2}(v)\right|=n_{k}$. Clearly $|V(T)|=n=1+k+n_{k}$. By Lemma 2.3,

$$
\sum_{\{y, x\} \subseteq N_{2}(v)} d(y, x) \leq n_{k}\left(2 n_{k}-\frac{n_{k}}{k}-1\right) .
$$

It is easy to check that

$$
\sum_{\{y, x\} \subseteq N(v)} d(y, x)=2\left(\begin{array}{l}
k \\
2
\end{array}\right), \quad \sum_{y \in N_{2}(v)} \sum_{x \in N(v)} d(y, x)=n_{k}(1+3(k-1))
$$

and

$$
\sum_{x \in V(T)} d(v, x)=k+2 n_{k}
$$

Consequently,

$$
\begin{aligned}
W(T) & =\sum_{\{y, x\} \subseteq N_{2}(v)} d(y, x)+\sum_{\{y, x\} \subseteq N(v)} d(y, x)+\sum_{y \in N_{2}(v)} \sum_{x \in N(v)} d(y, x)+\sum_{x \in V(T)} d(v, x) \\
& \leq 2 n_{k}^{2}-\frac{n_{k}^{2}}{k}+(3 k-1) n_{k}+k^{2} \\
& =2 n^{2}-\frac{(n-1)^{2}}{k}-k n-3 n+k+1=f(k) .
\end{aligned}
$$

Then the derivative $f^{\prime}(k)=0$ yields the value $k=\sqrt{n-1}$, which gives us the maximum of $f(k)$. It follows that

$$
W(T) \leq 2 n^{2}-2 n \sqrt{n-1}-3 n+2 \sqrt{n-1}+1 .
$$


Note that our bound is best possible. If every vertex in $N[v]$ is of degree $\sqrt{n-1}$, where $n-1$ is a square, then by Lemma 2.3 we have equality in (3.1), and consequently equality in (3.2) as well.

THEOREM 3.3. Let $T$ be a tree of order $n$ and diameter 5 . Then the Wiener index

$$
W(T) \leq \frac{9 n^{2}}{4}-2 n^{3 / 2}+O(n)
$$

and the bound is best possible.

Proof. Let $T$ be a tree with the maximal Wiener index among all trees of order $n$ and diameter 5. We denote the central vertices of $T$ by $v$ and $u$. Let $K_{1}=N(v) \backslash\{u\}$, $L_{1}=N(u) \backslash\{v\}$, and let $K_{2}\left(L_{2}\right)$ contain every leaf which has a neighbour in $K_{1}$ (in $L_{1}$ ). Clearly $V(T)=\{v, u\} \cup K_{1} \cup L_{1} \cup K_{2} \cup L_{2}$. Let $\left|K_{1}\right|=k,\left|L_{1}\right|=l,\left|K_{3}\right|=n_{k}$ and $\left|L_{3}\right|=n_{l}$.

Claim 1. We show that

$$
\begin{gathered}
W(T) \leq 2\left(n_{k}+n_{l}\right)^{2}+n_{k} n_{l}-\frac{n_{k}^{2}}{k}-\frac{n_{l}^{2}}{l}+3(k+l)\left(n_{k}+n_{l}\right) \\
+l n_{k}+k n_{l}+k^{2}+l^{2}+3 k l+2 k+2 l+1 .
\end{gathered}
$$

From Corollary 2.4 it follows that

$$
\sum_{\{x, y\} \subseteq K_{2}} d(x, y) \leq n_{k}\left(2 n_{k}-\frac{n_{k}}{k}-1\right) \quad \text { and } \sum_{\{x, y\} \subseteq L_{2}} d(x, y) \leq n_{l}\left(2 n_{l}-\frac{n_{l}}{l}-1\right) .
$$

It can be checked that

$$
\begin{aligned}
\sum_{x \in K_{2}} \sum_{y \in K_{1} \cup L_{1} \cup\{v, u\}} d(x, y) & =n_{k}(1+2+3 k+4 l), \\
\sum_{x \in L_{2}} \sum_{y \in K_{1} \cup L_{1} \cup\{v, u\}} d(x, y) & =n_{l}(1+2+3 l+4 k), \\
\sum_{x \in K_{2}} \sum_{y \in L_{2}} d(x, y) & =5 n_{k} n_{l}, \quad \sum_{x \in K_{1}} \sum_{y \in L_{1}} d(x, y)=3 k l, \\
\sum_{\{x, y\} \subseteq K_{1}} d(x, y) & =2\left(\begin{array}{l}
k \\
2
\end{array}\right)=k(k-1), \quad \sum_{\{x, y\} \subseteq L_{1}} d(x, y)=2\left(\begin{array}{l}
l \\
2
\end{array}\right)=l(l-1), \\
\sum_{x \in K_{1} \cup L_{1}} d(v, x) & =k+2 l, \sum_{x \in K_{1} \cup L_{1}} d(u, x)=l+2 k \quad \text { and } \quad d(u, v)=1 .
\end{aligned}
$$

Hence

$$
\begin{gathered}
W(T) \leq 2\left(n_{k}+n_{l}\right)^{2}+n_{k} n_{l}-\frac{n_{k}^{2}}{k}-\frac{n_{l}^{2}}{l}+3(k+l)\left(n_{k}+n_{l}\right) \\
+\ln _{k}+k n_{l}+k^{2}+l^{2}+3 k l+2 k+2 l+1 .
\end{gathered}
$$


By Lemma 2.5(i), if $k \geq 2$ (if $l \geq 2$ ), then $|N(x)|+|N(y)|>\sqrt{2 n}-1$ for any two vertices $x, y$ in $K_{1}$ (in $L_{1}$ ). Since by Corollary $2.2|N(x)|$ and $|N(y)|$ differ by at most 1 , both $|N(x)|$ and $|N(y)|$ are greater than $\sqrt{n / 2-1}$. By Lemma 2.5(ii), $|N(x)|,|N(y)|<\sqrt{2 n}+1$. Hence if $k \geq 2$ (if $l \geq 2$ ) then we can assume that every vertex in $K_{1}$ (in $L_{1}$ ) is adjacent to $c_{1} \sqrt{n}+O(1)$ vertices in $K_{2}$, where $\sqrt{2} / 2 \leq c_{1} \leq \sqrt{2}$ (to $c_{2} \sqrt{n}+O(1)$ vertices in $\left.L_{2}, \sqrt{2} / 2 \leq c_{2} \leq \sqrt{2}\right)$. It follows that $n_{k}=k\left(c_{1} \sqrt{n}+O(1)\right)$ and $n_{l}=l\left(c_{2} \sqrt{n}+O(1)\right)$, and consequently $k \leq \sqrt{n} / c_{1}+O(1)$ and $l \leq \sqrt{n} / c_{2}+O(1)$ (since $n_{k}$ and $n_{l}$ cannot exceed $\left.n\right)$.

Claim 2. We have $n_{k}=n_{l}+O\left(n^{1 / 2}\right)$.

Suppose to the contrary that $n_{k}>n_{l}+O\left(n^{1 / 2}\right)$. Let $w$ be any vertex in $K_{2}$, let $v_{1}$ be the neighbour of $w$ in $T\left(v_{1} \in K_{1}\right)$, and let $u_{1}$ be any vertex in $L_{1}$. Let $T^{\prime}=T-v_{1} w+u_{1} w$. We have $d\left(T^{\prime}\right)=d(T)=5$,

$$
\begin{aligned}
\sum_{w^{\prime} \in V(T)} d\left(w, w^{\prime}\right) & =1+2\left(c_{1} \sqrt{n}+O(1)\right)+3 k+4\left(n_{k}-c_{1} \sqrt{n}-O(1)\right)+4 l+5 n_{l} \\
& =4 n_{k}+5 n_{l}+O\left(n^{1 / 2}\right)
\end{aligned}
$$

and

$$
\sum_{w^{\prime} \in V\left(T^{\prime}\right)} d\left(w, w^{\prime}\right)=4 n_{l}+5 n_{k}+O\left(n^{1 / 2}\right)
$$

Then

$$
0 \leq W(T)-W\left(T^{\prime}\right)=\sum_{w^{\prime} \in V(T)} d\left(w, w^{\prime}\right)-\sum_{w^{\prime} \in V\left(T^{\prime}\right)} d\left(w, w^{\prime}\right)=n_{l}-n_{k}+O\left(n^{1 / 2}\right),
$$

which is a contradiction.

Analogously it can be shown that $n_{l}$ cannot be greater than $n_{k}+O\left(n^{1 / 2}\right)$.

Since $n=n_{k}+n_{l}+k+l+2=n_{k}+n_{l}+O\left(n^{1 / 2}\right)$, we have $n_{k}=n_{l}=n / 2+O\left(n^{1 / 2}\right)$. We can write $n_{k}=n / 2+c_{1}^{\prime} \sqrt{n}+O(1)$ and $n_{l}=n / 2+c_{2}^{\prime} \sqrt{n}+O(1)$, where $c_{1}^{\prime}$ and $c_{2}^{\prime}$ are real numbers.

We also know that $n_{k}=k\left(c_{1} \sqrt{n}+O(1)\right)$ which implies that $k=\sqrt{n} / 2 c_{1}+O(1)$. Similarly we obtain $l=\sqrt{n} / 2 c_{2}+O(1)$.

By Claim 1,

$$
W(T) \leq 2\left(n_{k}+n_{l}\right)^{2}+n_{k} n_{l}-\frac{n_{k}^{2}}{k}-\frac{n_{l}^{2}}{l}+3(k+l)\left(n_{k}+n_{l}\right)+l n_{k}+k n_{l}+O(n),
$$

and from the previous part of the proof it follows that

$$
\begin{gathered}
\left(n_{k}+n_{l}\right)^{2}=(n-k-l-2)^{2}=n^{2}-2 k n-2 l n+O(n), \\
\frac{n_{k}^{2}}{k}=\left(\frac{n}{2}+c_{1}^{\prime} \sqrt{n}+O(1)\right)^{2} / k=\frac{n^{2}}{4 k}+O(n), \quad \frac{n_{l}^{2}}{l}=\frac{n^{2}}{4 l}+O(n), \\
(k+l)\left(n_{k}+n_{l}\right)=(k+l) n+O(n), \quad k n_{l}+\ln _{k}=(k+l) \frac{n}{2}+O(n) .
\end{gathered}
$$


Since

$$
n=n_{k}+n_{l}+k+l+2=\left(\frac{n}{2}+c_{1}^{\prime} \sqrt{n}\right)+\left(\frac{n}{2}+c_{2}^{\prime} \sqrt{n}\right)+k+l+O(1),
$$

we obtain $\left(c_{1}^{\prime}+c_{2}^{\prime}\right) \sqrt{n}=-k-l+O(1)$. Consequently,

$$
n_{k} n_{l}=\frac{n^{2}}{4}+\left(c_{1}^{\prime}+c_{2}^{\prime}\right) \sqrt{n} \frac{n}{2}+O(n)=\frac{n^{2}}{4}-(k+l) \frac{n}{2}+O(n) .
$$

It follows that

$$
W(T) \leq \frac{9 n^{2}}{4}-(k+l) n-\frac{n^{2}}{4}\left(\frac{1}{k}+\frac{1}{l}\right)+O(n)=f(k, l) .
$$

Then the partial derivatives $f_{k}(k, l)=0$ and $f_{l}(k, l)=0$ show that $f(k, l)$ is maximised for $k=l=\sqrt{n} / 2$. Hence

$$
W(T) \leq \frac{9 n^{2}}{4}-2 n^{3 / 2}+O(n) .
$$

It can be checked that if $\left|K_{1}\right|=\left|L_{1}\right|=\sqrt{n-2} / 2$ and every vertex in $K_{1}$ and $L_{1}$ is adjacent to $\sqrt{n-2}-1$ leaves, where $n-2$ is a power of 4 , then $W(T)=9 n^{2} / 4-$ $2 n^{3 / 2}+O(n)$. The proof is complete.

Theorem 3.4. Let $T$ be a tree of order $n$ and diameter 6 . Then

$$
W(T) \leq 3 n^{2}-2 \sqrt{6} n^{3 / 2}-2 n+O\left(n^{1 / 2}\right)
$$

and the bound is best possible.

Proof. Let $T$ be a tree with the maximal Wiener index among all trees of order $n$ and diameter 6 . We denote the central vertex of $T$ by $v$.

Note that instead of Claims 1 and 2 one could prove a more general claim saying that all leaves of $T$ must be at distance 3 from $v$. However, we do not need such a result to prove our theorem.

Claim 1. There is no leaf joined to $v$.

Suppose to the contrary that $x$ is a leaf joined to $v$. Since $v$ is the central vertex of a tree of diameter 6 , there must be at least two other vertices $u_{1}, u_{2}$ adjacent to $v$ in $T$. Let $U_{i}$ be the set which contains all vertices $u$ that satisfy the inequality $d_{T}\left(u, u_{i}\right)<d_{T}(u, v)$, $i=1$, 2. Then $U_{1} \cap U_{2}=\emptyset$. Since $\left|U_{1}\right|+\left|U_{2}\right| \leq n-2$, at least one set $U_{i}$ contains at most $n / 2-1$ vertices. Without loss of generality, we can suppose that $\left|U_{1}\right| \leq n / 2-1$. Let $T^{\prime}=T-v x+u_{1} x$. Then $d\left(T^{\prime}\right)=d(T)=6$ and

$$
W\left(T^{\prime}\right)-W(T)=\sum_{u \in V(T)}\left(d_{T^{\prime}}(x, u)-d_{T}(x, u)\right) .
$$

Since $d_{T^{\prime}}(x, u)=d_{T}(x, u)-1$ for any $u \in U_{1}$, and $d_{T^{\prime}}\left(x, u^{\prime}\right)=d_{T}\left(x, u^{\prime}\right)+1$ for any $u^{\prime} \in V(T) \backslash\left(U_{1} \cup\{x\}\right)$, we get $W\left(T^{\prime}\right)>W(T)$. 
Claim 2. The vertex $v$ does not have a neighbour of degree two which is adjacent to a leaf.

Suppose that $v$ has a neighbour $x_{1}$ of degree two which is adjacent to a leaf, say $x_{2}$. As in the previous claim, one can show that there must be a neighbour of $v$, say $u_{1}$, such that $d_{T}\left(u_{1}, u\right)<d_{T}(v, u)$ for at most $(n-3) / 2$ vertices $u$ of $T$. Then for $T^{\prime}=T-v x_{1}+u_{1} x_{1}$ we get $d_{T^{\prime}}\left(x_{i}, u\right)=d_{T}\left(x_{1}, u\right)-1$ for at most $(n-3) / 2$ vertices $u$, and $d_{T^{\prime}}\left(x_{i}, u^{\prime}\right)=d_{T}\left(x_{i}, u^{\prime}\right)+1$ for at least $(n-1) / 2$ vertices $u^{\prime}(i=1,2)$. Consequently,

$$
W\left(T^{\prime}\right)-W(T)=2 \sum_{u \in V(T)}\left(d_{T^{\prime}}\left(x_{1}, u\right)-d_{T}\left(x_{1}, u\right)\right) \geq 2 .
$$

Claim 3. Each neighbour of $v$ has degree at most three.

Suppose to the contrary that $v_{1}$ is a neighbour of $v$, which is adjacent to at least three other vertices $v_{2}, v_{2}^{\prime}$ and $v_{2}^{\prime \prime}$. Let $V_{3}\left(V_{3}^{\prime}, V_{3}^{\prime \prime}\right)$ be the set of leaves adjacent to $v_{2}\left(v_{2}^{\prime}, v_{2}^{\prime \prime}\right)$. Without loss of generality, we can assume that $\left|V_{3}\right| \geq\left|V_{3}^{\prime \prime}\right| \geq\left|V_{3}^{\prime}\right| \geq 0$. Let

$$
T^{\prime}=T-\bigcup_{w \in V_{3}^{\prime}} w v_{2}^{\prime}-v_{1} v_{2}^{\prime}+\bigcup_{w \in V_{3}^{\prime}} v_{2} w+v_{2} v_{2}^{\prime} .
$$

Analogous steps as the ones in the proof of Lemma 2.5(i) yield

$$
W\left(T^{\prime}\right)-W(T)=n-2\left|V_{3}\right|\left|V_{3}^{\prime}\right|-2\left|V_{3}^{\prime}\right|-2\left|V_{3}^{\prime}\right|-3,
$$

(see (2.1)). Note that if $\left|V_{3}\right|=\left|V_{3}^{\prime}\right|=0$, then $W\left(T^{\prime}\right)-W(T)>0$, so we can assume that there is a vertex, say $v_{3} \in V_{3}$.

Let $T^{\prime \prime}=T-v_{1} v_{2}+v v_{3}$. Then $d_{T}\left(w_{1}, w_{2}\right) \neq d_{T^{\prime \prime}}\left(w_{1}, w_{2}\right)$ in the following cases:

$$
\begin{gathered}
d_{T^{\prime \prime}}\left(w_{1}, w_{2}\right)=d_{T}\left(w_{1}, w_{2}\right)+2 \quad \text { if } w_{1} \in V_{3} \cup\left\{v_{2}\right\} \backslash\left\{v_{3}\right\}, w_{2} \in V_{3}^{\prime} \cup V_{3}^{\prime \prime} \cup\left\{v_{1}, v_{2}^{\prime}, v_{2}^{\prime \prime}\right\} \\
d_{T^{\prime \prime}}\left(v_{3}, w\right)=d_{T}\left(v_{3}, w\right)-2 \quad \text { if } w \in V(T) \backslash\left(V_{3} \cup V_{3}^{\prime} \cup V_{3}^{\prime \prime} \cup\left\{v_{1}, v_{2}, v_{2}^{\prime}, v_{2}^{\prime \prime}\right\}\right) .
\end{gathered}
$$

Consequently,

$$
\begin{aligned}
W(T)-W\left(T^{\prime \prime}\right) & =-2\left|V_{3}\right|\left(\left|V_{3}^{\prime}\right|+\left|V_{3}^{\prime \prime}\right|+3\right)+2\left(n-\left|V_{3}\right|-\left|V_{3}^{\prime}\right|-\left|V_{3}^{\prime \prime}\right|-4\right) \\
& =2\left(n-\left|V_{3}\right|\left|V_{3}^{\prime}\right|-\left|V_{3}\right|\left|V_{3}^{\prime \prime}\right|-4\left|V_{3}\right|-\left|V_{3}^{\prime}\right|-\left|V_{3}^{\prime \prime}\right|-4\right) .
\end{aligned}
$$

Since $T$ has the maximum Wiener index among all graphs of order $n$ and diameter $d$, we have $W\left(T^{\prime}\right)-W(T) \leq 0 \leq\left(W(T)-W\left(T^{\prime \prime}\right)\right) / 2$ which yields

$$
\begin{aligned}
0 & \leq\left|V_{3}\right|\left|V_{3}^{\prime}\right|-\left|V_{3}\right|\left|V_{3}^{\prime \prime}\right|-2\left|V_{3}\right|+\left|V_{3}^{\prime}\right|-\left|V_{3}^{\prime \prime}\right|-1 \\
& =\left(\left|V_{3}\right|+1\right)\left(\left|V_{3}^{\prime}\right|-\left|V_{3}^{\prime \prime}\right|\right)-2\left|V_{3}\right|-1 .
\end{aligned}
$$

Since $\left|V_{3}^{\prime}\right| \leq\left|V_{3}^{\prime \prime}\right|$ and $\left|V_{3}\right| \geq 1$, we get a contradiction.

Let $K_{1}\left(L_{1}\right)$ be the set of neighbours of $v$ which are of degree two (of degree three), and let $K_{i}\left(L_{i}\right)$ be the set of vertices at distance $i$ from $v$, such that every vertex in $K_{i}$ (in $L_{i}$ ) has a neighbour in $K_{i-1}$ (in $\left.L_{i-1}\right), i=2,3$. Let $\left|K_{1}\right|=k,\left|L_{1}\right|=l,\left|K_{3}\right|=n_{k}$ and $\left|L_{3}\right|=n_{l}$. Clearly $n=1+2 k+3 l+n_{k}+n_{l}$. 
Claim 4. For any two vertices $v_{2}$ and $v_{2}^{\prime}$ in $K_{2}$, where $V_{3}\left(V_{3}^{\prime}\right)$ is the set of neighbours of $v_{2}$ (of $v_{2}^{\prime}$ ) in $K_{3}$, we have $\left|V_{3}\right|+\left|V_{3}^{\prime}\right|>\sqrt{3 n}-5$.

Let $v_{1}\left(v_{1}^{\prime}\right)$ be the vertex in $K_{1}$ adjacent to $v_{2}$ (to $v_{2}^{\prime}$ ), and let $V_{3}\left(V_{3}^{\prime}\right)$ be the set of leaves adjacent to $v_{2}$ (to $v_{2}^{\prime}$ ). Let

$$
T^{\prime}=T-\bigcup_{w \in V_{3}^{\prime}} w v_{2}^{\prime}-v v_{1}^{\prime}-v_{1}^{\prime} v_{2}^{\prime}+\bigcup_{w \in V_{3}^{\prime}} v_{2} w+v_{2} v_{1}^{\prime}+v_{2} v_{2}^{\prime}
$$

We mention all cases when $d_{T}\left(w_{1}, w_{2}\right) \neq d_{T^{\prime \prime}}\left(w_{1}, w_{2}\right)$. We have

$$
\begin{aligned}
d_{T^{\prime}}\left(w_{1}, w_{2}\right) & =d_{T}\left(w_{1}, w_{2}\right)-4 \quad \text { if } w_{1} \in V_{3} \cup\left\{v_{2}\right\}, w_{2} \in V_{3}^{\prime}, \\
d_{T^{\prime}}\left(v_{2}^{\prime}, w\right) & =d_{T}\left(v_{2}^{\prime}, w\right)-3 \quad \text { if } w \in V_{3} \cup\left\{v_{2}\right\}, \\
d_{T^{\prime}}\left(v_{1}^{\prime}, w\right) & =d_{T}\left(v_{1}^{\prime}, w\right)-2 \quad \text { if } w \in V_{3} \cup\left\{v_{2}\right\}, \\
d_{T^{\prime}}\left(v_{1}, w\right) & =d_{T}\left(v_{1}, w\right)-2 \quad \text { if } w \in V_{3}^{\prime}, \\
d_{T^{\prime}}\left(v_{1}, v_{2}^{\prime}\right) & =d_{T}\left(v_{1}, v_{2}^{\prime}\right)-1, \\
d_{T^{\prime}}\left(v_{2}^{\prime}, w\right) & =d_{T}\left(v_{2}^{\prime}, w\right)+1 \quad \text { if } w \in V(G) \backslash\left(V_{3} \cup\left\{v_{1}, v_{2}, v_{2}^{\prime}\right\}\right) \\
d_{T^{\prime}}\left(v_{1}^{\prime}, w\right) & =d_{T}\left(v_{1}^{\prime}, w\right)+2 \quad \text { if } w \in V(G) \backslash\left(V_{3} \cup V_{3}^{\prime} \cup\left\{v_{1}, v_{1}^{\prime}, v_{2}, v_{2}^{\prime}\right\}\right) .
\end{aligned}
$$

Then

$$
\begin{aligned}
W\left(T^{\prime}\right)-W(T)= & 2\left(n-\left|V_{3}\right|-\left|V_{3}^{\prime}\right|-4\right)+\left(n-\left|V_{3}\right|-3\right) \\
& -1-2\left(\left|V_{3}\right|+\left|V_{3}^{\prime}\right|+1\right)-3\left(\left|V_{3}\right|+1\right)-4\left(\left|V_{3}\right|+1\right)\left|V_{3}^{\prime}\right| \\
= & 3 n-4\left|V_{3}\right|\left|V_{3}^{\prime}\right|-8\left|V_{3}\right|-8\left|V_{3}^{\prime}\right|-17 \\
= & 3 n-4\left(\frac{\left|V_{3}\right|+\left|V_{3}^{\prime}\right|}{2}\right)^{2}-8\left(\left|V_{3}\right|+\left|V_{3}^{\prime}\right|\right)-17 .
\end{aligned}
$$

If $\left|V_{3}\right|+\left|V_{3}^{\prime}\right| \leq \sqrt{3 n}-5$, then we get $W\left(T^{\prime}\right)-W(T) \geq 2(\sqrt{3 n}-1)>0$. It can be checked that $d\left(T^{\prime}\right) \leq d(T)$. If $d\left(T^{\prime}\right)<d(T)$, it is easy to transform $T^{\prime}$ to $T^{\prime \prime}$ such that $V\left(T^{\prime \prime}\right)=V(T), d\left(T^{\prime \prime}\right)=d(T)$ and $W\left(T^{\prime \prime}\right)>W\left(T^{\prime}\right)>W(T)$. So $W(T)$ is not the maximum Wiener index of trees of order $n$ and diameter 6.

Claim 5. We have $l<\sqrt{n / 2}$ and $k<\sqrt{3 n}$.

By Claim 4, for the sets of neighbours $V_{3}$ and $V_{3}^{\prime}$ of any two vertices $v_{2}$ and $v_{2}^{\prime}$ in $K_{2}$ we have $\left|V_{3}\right|+\left|V_{3}^{\prime}\right|>\sqrt{3 n}-5$. If $k$ is even, then $n_{k}>(k / 2)(\sqrt{3 n}-5)$. From Corollary 2.2 we know that $\left|V_{3}\right|$ and $\left|V_{3}^{\prime}\right|$ differ by at most 1 , so the number of leaves joined to any vertex in $K_{2}$ is greater than $\sqrt{3 n} / 2-3$. Hence, if $k$ is odd,

$$
n_{k}>\frac{k-1}{2}(\sqrt{3 n}-5)+\frac{\sqrt{3 n}}{2}-3=\frac{k}{2}(\sqrt{3 n}-5)-\frac{1}{2} .
$$

Then $n>1+2 k+(k / 2)(\sqrt{3 n}-5)-1 / 2$ which implies that $k<(2 n-1) /(\sqrt{3 n}-1)<$ $(2+\epsilon) n / \sqrt{3 n}$ for some small $\epsilon>0$. For us it suffices to use $\epsilon=1$. 
By Lemma 2.5(i), if $v_{2}$ and $v_{2}^{\prime}$ are any two vertices in $L_{2}$ which have a common neighbour, where $V_{3}\left(V_{3}^{\prime}\right)$ is the set of neighbours of $v_{2}$ (of $v_{2}^{\prime}$ ) in $L_{3}$, then $\left|V_{3}\right|+$ $\left|V_{3}^{\prime}\right|>\sqrt{2 n}-3$. We get $n_{l}>(\sqrt{2 n}-3) l$ which yields $n>1+3 l+(\sqrt{2 n}-3) l$, and consequently $l<\sqrt{n / 2}$.

Claim 6. Let $v_{1}, u_{1} \in L_{1}$ and let $V_{3}\left(U_{3}\right)$ be a subset of $L_{3}$ containing vertices which are at distance 2 from $v_{1}\left(u_{1}\right)$. Then $\left|V_{3}\right|$ and $\left|U_{3}\right|$ differ by at most 1 .

Suppose that $\left|V_{3}\right| \geq\left|U_{3}\right|+2$. Let $v_{2}^{\prime}, v_{2}^{\prime \prime}\left(u_{2}^{\prime}, u_{2}^{\prime \prime}\right)$ be two vertices in $L_{2}$ adjacent to $v_{1}\left(u_{1}\right)$, and let $V_{3}^{\prime}\left(V_{3}^{\prime \prime}, U_{3}^{\prime}, U_{3}^{\prime \prime}\right)$ be the set of neighbours of $v_{2}^{\prime}\left(v_{2}^{\prime \prime}, u_{2}^{\prime}, u_{2}^{\prime \prime}\right)$ in $L_{3}$. Since $\left|V_{3}^{\prime}\right|+\left|V_{3}^{\prime \prime}\right| \geq\left|U_{3}^{\prime}\right|+\left|U_{3}^{\prime \prime}\right|+2$, without loss of generality we can assume that $\left|V_{3}^{\prime}\right| \geq$ $\left|U_{3}^{\prime}\right|+1$. Let $w$ be any vertex in $V_{3}^{\prime}$ and let $T^{\prime}=T-v_{2}^{\prime} w+u_{2}^{\prime} w$. Since $\left|N_{2}^{T}\left(v_{1}\right) \cap L_{3}\right| \geq 2$, we have $\left|N_{2}^{T^{\prime}}\left(v_{1}\right) \cap L_{3}\right| \geq 1$, which implies that there must be two vertices at distance 6 in $T^{\prime}$. Hence $d(T)=d\left(T^{\prime}\right)$. It can be checked that $d_{T}\left(w_{1}, w_{2}\right)=d_{T^{\prime}}\left(w_{1}, w_{2}\right)$ for any two vertices $w_{1}, w_{2}$ different from $w$, and $\left|N_{i}^{T}(w)\right|=\left|N_{i}^{T^{\prime}}(w)\right|$ if $i=1,3,5$. We have

$$
\begin{aligned}
& \left|N_{2}^{T^{\prime}}(w)\right|-\left|N_{2}^{T}(w)\right|=\left(\left|U_{3}^{\prime}\right|+1\right)-\left|V_{3}^{\prime}\right|, \\
& \left|N_{4}^{T^{\prime}}(w)\right|-\left|N_{4}^{T}(w)\right|=\left(\left|U_{3}^{\prime \prime}\right|+1\right)-\left(\left|V_{3}^{\prime \prime}\right|+1\right), \\
& \left|N_{6}^{T^{\prime}}(w)\right|-\left|N_{6}^{T}(w)\right|=\left(\left|V_{3}\right|-1\right)-\left|U_{3}\right| .
\end{aligned}
$$

Then

$$
\begin{aligned}
W\left(T^{\prime}\right)-W(T) & =\sum_{i=1}^{6} i\left(\left|N_{i}^{T^{\prime}}(w)\right|-\left|N_{i}^{T}(w)\right|\right) \\
& =6\left(\left|V_{3}\right|-\left|U_{3}\right|\right)-4\left(\left|V_{3}^{\prime \prime}\right|-\left|U_{3}^{\prime \prime}\right|\right)-2\left(\left|V_{3}^{\prime}\right|-\left|U_{3}^{\prime}\right|\right)-4 \\
& =2\left(\left|V_{3}\right|-\left|U_{3}\right|-2\right)+2\left(\left|V_{3}^{\prime}\right|-\left|U_{3}^{\prime}\right|\right) \geq 2\left(\left|V_{3}^{\prime}\right|-\left|U_{3}^{\prime}\right|\right)>0,
\end{aligned}
$$

which is a contradiction.

Claim 7. We have

$$
\sum_{\{x, y\} \subseteq L_{3}} d(x, y) \leq n_{l}\left(3 n_{l}-\frac{3 n_{l}}{2 l}-1\right) .
$$

Let $L_{1}=\left\{v_{1}, v_{2}, \ldots, v_{l}\right\}$ and let $v, u_{i}, w_{i}$ be the neighbours of $v_{i}, i=1,2, \ldots, l$. By Claim 6, the number of vertices in $L_{3}$ which are at distance 2 from $v_{i}$ is either $2 s$ or $2 s+\epsilon$, where $s$ is an integer, and $\epsilon=1$ or -1 . Without loss of generality, we can assume that the number of vertices in $L_{3}$ which are at distance 2 from $v_{j}$ $(j=1,2, \ldots, p, 0 \leq p \leq l)$ is $2 s+\epsilon$, and the number of vertices in $L_{3}$ which are at distance 2 from $v_{j}(j=p+1, p+2, \ldots, l)$ is $2 s$. Then by Corollary 2.2 we can assume that $u_{i}(i=1,2, \ldots, l)$ and $w_{j}(j=p+1, p+2, \ldots, l)$ are adjacent to $s$ vertices in $L_{3}$, and $w_{j}(j=1,2, \ldots, p)$ are adjacent to $s+\epsilon$ vertices in $L_{3}$. It follows that

$$
\left|L_{3}\right|=n_{l}=(2 l-p) s+p(s+\epsilon)=2 l s+\epsilon p .
$$


Then, for the vertices $w, w^{\prime}, w^{\prime \prime}$ in $L_{3}$,

$$
\begin{gathered}
\sum_{v^{\prime} \in L_{3}} d\left(w, v^{\prime}\right)=2(s-1)+4 s+6(2 l s+\epsilon p-2 s) \\
\quad \text { if } w \in N\left(u_{j}\right) \cup N\left(w_{j}\right), j=p+1, p+2, \ldots, l, \\
\sum_{v^{\prime} \in L_{3}} d\left(w^{\prime}, v^{\prime}\right)=2(s-1)+4(s+\epsilon)+6(2 l s+\epsilon p-2 s-\epsilon) \\
\quad \text { if } w^{\prime} \in N\left(u_{i}\right), i=1,2, \ldots, p \\
\sum_{v^{\prime} \in L_{3}} d\left(w^{\prime \prime}, v^{\prime}\right)=2(s+\epsilon-1)+4 s+6(2 l s+\epsilon p-2 s-\epsilon) \\
\text { if } w^{\prime \prime} \in N\left(w_{i}\right), i=1,2, \ldots, p,
\end{gathered}
$$

which yield

$$
\begin{aligned}
2 \sum_{\left\{v^{\prime \prime}, v^{\prime}\right\} \subseteq L_{3}} d\left(v^{\prime \prime}, v^{\prime}\right)= & \sum_{v^{\prime \prime} \in L_{3}} \sum_{v^{\prime} \in L_{3}} d\left(v^{\prime \prime}, v^{\prime}\right) \\
= & 2(l-p) s \sum_{v^{\prime} \in L_{3}} d\left(w, v^{\prime}\right)+p s \sum_{v^{\prime} \in L_{3}} d\left(w^{\prime}, v^{\prime}\right) \\
& \quad+p(s+\epsilon) \sum_{v^{\prime} \in L_{3}} d\left(w^{\prime \prime}, v^{\prime}\right) \\
= & (2 l s+\epsilon p)(6(2 l s+\epsilon p)-6 s-2)-p(6 \epsilon s+4) .
\end{aligned}
$$

Since $p / l \leq 1$, we have $-p(6 \epsilon s+4) \leq-p(6 \epsilon s+3 p / l)=-(3 \epsilon p / l)(2 l s+\epsilon p)$. Consequently,

$$
\sum_{\{w, u\} \subseteq L_{3}} d(w, u) \leq \frac{2 l s+\epsilon p}{2}\left(6(2 l s+\epsilon p)-6 s-\frac{3 \epsilon p}{l}-2\right)=\frac{n_{l}}{2}\left(6 n_{l}-\frac{3 n_{l}}{l}-2\right) .
$$

Claim 8. We have

$$
\begin{array}{r}
W(T) \leq 3\left(n_{k}+n_{l}\right)^{2}+(9 k+14 l)\left(n_{k}+n_{l}\right)-\frac{2 n_{k}^{2}}{k}-\frac{3 n_{l}^{2}}{2 l} \\
-4 n_{k}-6 n_{l}+6 k^{2}+15 l^{2}+19 k l-2 k-6 l .
\end{array}
$$

It can be checked that

$$
\begin{aligned}
\sum_{u \in K_{3}} \sum_{w \in L_{3}} d(u, w) & =6 n_{k} n_{l}, \\
\sum_{u \in K_{3}} \sum_{w \in V(G) \backslash\left(K_{3} \cup L_{3}\right)} d(u, w) & =n_{k}(1+2+3+4(k-1)+4 l+5(k-1)+5 \cdot 2 l) \\
& =n_{k}(9 k+14 l-3),
\end{aligned}
$$




$$
\begin{aligned}
\sum_{u \in L_{3}} \sum_{w \in V(G) \backslash\left(K_{3} \cup L_{3}\right)} d(u, w) & =n_{l}(1+2+3 \cdot 2+4 k+4(l-1)+5 k+5 \cdot 2(l-1)) \\
& =n_{l}(9 k+14 l-5), \\
\sum_{\{u, w\} \subseteq K_{2}} d(u, w) & =4\left(\begin{array}{l}
k \\
2
\end{array}\right)=2 k(k-1), \\
\sum_{\{u, w\} \subseteq L_{2}} d(u, w) & =l(8 l-6) \text { since for any } u \in L_{2}, \\
\sum_{w \in L_{2}} d(u, w) & =2+4 \cdot 2(l-1), \\
\sum_{\sum_{u \in K_{2}}} d(u, w) & =k(1+2+3(k-1)+3 l+4 \cdot 2 l)=k(3 k+11 l), \\
\sum_{u \in L_{2}} \sum_{w \in K_{1} \cup L_{1} \cup\{v\}} d(u, w) & =2 l(1+2+3 k+3(l-1))=6 l(l+k) .
\end{aligned}
$$

Finally,

$$
\begin{gathered}
\sum_{\{u, w\} \subseteq K_{1}} d(u, w)=2\left(\begin{array}{l}
k \\
2
\end{array}\right)=k(k-1), \quad \sum_{\{u, w\} \subseteq L_{1}} d(u, w)=2\left(\begin{array}{l}
l \\
2
\end{array}\right)=l(l-1), \\
\sum_{u \in K_{1}} \sum_{w \in L_{1}} d(u, w)=2 k l, \quad \sum_{w \in K_{1} \cup L_{1}} d(v, w)=k+l .
\end{gathered}
$$

By Claim 7,

$$
\sum_{\{x, y\} \subseteq L_{3}} d(x, y) \leq n_{l}\left(3 n_{l}-\frac{3 n_{l}}{2 l}-1\right)
$$

and from Corollary 2.4 it follows that

$$
\sum_{\{x, y\} \subseteq K_{3}} d(x, y) \leq n_{k}\left(3 n_{k}-\frac{2 n_{k}}{k}-1\right) .
$$

Hence,

$$
\begin{array}{r}
W(T) \leq 3\left(n_{k}+n_{l}\right)^{2}+(9 k+14 l)\left(n_{k}+n_{l}\right)-\frac{2 n_{k}^{2}}{k}-\frac{3 n_{l}^{2}}{2 l} \\
-4 n_{k}-6 n_{l}+6 k^{2}+15 l^{2}+19 k l-2 k-6 l
\end{array}
$$

Now we complete the proof of Theorem 3.4. Let

$$
\begin{array}{r}
f\left(n_{k}, n_{l}\right)=3\left(n_{k}+n_{l}\right)^{2}+(9 k+14 l)\left(n_{k}+n_{l}\right)-\frac{2 n_{k}^{2}}{k}-\frac{3 n_{l}^{2}}{2 l} \\
-4 n_{k}-6 n_{l}+6 k^{2}+15 l^{2}+19 k l-2 k-6 l .
\end{array}
$$

We find the maximum of $f\left(n_{k}, n_{l}\right)$ subject to the constraint

$$
n_{k}+n_{l}=n-2 k-3 l-1=a .
$$


Let $F\left(n_{k}, n_{l}, \lambda\right)=f\left(n_{k}, n_{l}\right)-\lambda\left(n_{k}+n_{l}-a\right)$. Then using $F_{n_{k}}\left(n_{k}, n_{l}, \lambda\right)=F_{n_{l}}\left(n_{k}, n_{l}, \lambda\right)$ we get $4 n_{k} / k=3 n_{l} / l-2$. Substituting $n_{l}=a-n_{k}$ yields $n_{k}=k(3 a-2 l) /(3 k+4 l)$, and then we obtain $n_{l}=2 l(2 a+k) /(3 k+4 l)$. It is easy to check that these values of $n_{k}$ and $n_{l}$ give the maximum of $F\left(n_{k}, n_{l}, \lambda\right)$. Hence $W(T)$ is at most

$$
\begin{aligned}
& 3 a^{2}+(9 k+14 l) a-\frac{2 k(3 a-2 l)^{2}}{(3 k+4 l)^{2}}-\frac{6 l(2 a+k)^{2}}{(3 k+4 l)^{2}}-\frac{4 k(3 a-2 l)}{3 k+4 l}-\frac{12 l(2 a+k)}{3 k+4 l} \\
& \quad+6 k^{2}+15 l^{2}+19 k l-2 k-6 l .
\end{aligned}
$$

Consequently,

$$
\frac{2 k(3 a-2 l)^{2}}{(3 k+4 l)^{2}}+\frac{6 l(2 a+k)^{2}}{(3 k+4 l)^{2}}=\frac{6 a^{2}+2 k l}{3 k+4 l}
$$

and

$$
\frac{4 k(3 a-2 l)}{3 k+4 l}+\frac{12 l(2 a+k)}{3 k+4 l}=\frac{12 a(k+2 l)+4 k l}{3 k+4 l},
$$

and, using $a=n-2 k-3 l-1$,

$$
\begin{aligned}
W(T) \leq 3 & n^{2}-(3 k+4 l) n-6 n+k-2 l+3 \\
& -\frac{6\left(n^{2}-2 k n-2 l n-2 n-3 l^{2}-k l+2 k+2 l+1\right)}{3 k+4 l} .
\end{aligned}
$$

Since by Claim 5, $k$ and $l$ are at most $O\left(n^{1 / 2}\right)$,

$$
\begin{aligned}
W(T) & \leq 3 n^{2}-(3 k+4 l) n-6 n-\frac{6 n(n-2 k-2 l-2)}{3 k+4 l}+O\left(n^{1 / 2}\right) \\
& =3 n^{2}-(3 k+4 l) n-6 n-\frac{6 n(n-2)}{3 k+4 l}+3 n\left(1+\frac{k}{3 k+4 l}\right)+O\left(n^{1 / 2}\right) .
\end{aligned}
$$

Let $b=3 k+4 l$ such that the expression above is maximal. Then

$$
3 n^{2}-b n-6 n-\frac{6 n(n-2)}{b}+3 n\left(1+\frac{k}{b}\right)
$$

is maximised for $b=3 k$ (and $l=0$ ). Now we need to find $b$ such that

$$
f(n, b)=3 n^{2}-(b+2) n-\frac{6 n(n-2)}{b}
$$

is maximal. The partial derivative $f_{b}(n, b)=0$ yields the value $b=\sqrt{6(n-2)}$, which gives us the maximum of $f(n, b)$, that is,

$$
\begin{aligned}
3 n^{2}-2 \sqrt{6(n-2)} n-2 n & \leq 3 n^{2}-2\left(\sqrt{6 n}-\frac{12}{\sqrt{6 n}}\right) n-2 n \\
& =3 n^{2}-2 \sqrt{6} n^{3 / 2}-2 n+O\left(n^{1 / 2}\right) .
\end{aligned}
$$

Clearly $W(T) \leq f(n, b)+O\left(n^{1 / 2}\right)$. 
It remains to prove that the upper bound is best possible. We show that there is an infinite family of trees $T_{1}$ such that

$$
W\left(T_{1}\right)=3 n^{2}-2 \sqrt{6} n^{3 / 2}-2 n+O\left(n^{1 / 2}\right) .
$$

Let $n=(3 / 2) k^{2}+1$ where $k$ is even. Let $T_{1}$ be a tree of order $n$, diameter 6 , with the central vertex $v$, where the degree of $v$ is $k$, any vertex in $N(v)$ has one neighbour in $N_{2}(v)$, and any vertex in $N_{2}(v)$ is adjacent to $n=(3 / 2) k-2=(1 / 2) \sqrt{6(n-1)}-2$ vertices in $N_{3}(v)$. Then $|N(v)|=\left|N_{2}(v)\right|=k=(1 / 3) \sqrt{6(n-1)}$ and

$$
\left|N_{3}(v)\right|=n_{k}=k\left(\frac{3}{2} k-2\right)=n-\frac{2}{3} \sqrt{6(n-1)}-1 .
$$

We have

$$
\sum_{\{y, x\} \subseteq N_{3}(v)} d(y, x)=n_{k}\left(3 n_{k}-\frac{2 n_{k}}{k}-1\right),
$$

which is the upper bound in Lemma 2.3 if the diameter is 6 . Consequently we get equality in Claim 8 (where in our case $l=0$ and $n_{l}=0$ ). It follows that

$$
W\left(T_{1}\right)=3 n_{k}^{2}+9 k n_{k}-\frac{2 n_{k}^{2}}{k}-4 n_{k}+6 k^{2}-2 k .
$$

Since $n_{k}=k(3 k / 2-2)$, we obtain $W\left(T_{1}\right)=(27 / 4) k^{4}-9 k^{3}+6 k^{2}-2 k$ or equivalently

$$
W\left(T_{1}\right)=3 n^{2}-2 n \sqrt{6(n-1)}-2 n+\frac{4}{3} \sqrt{6(n-1)}-1=3 n^{2}-2 \sqrt{6} n^{3 / 2}-2 n+O\left(n^{1 / 2}\right) .
$$

The proof is complete.

Since by (1.1) and (1.2), $D^{\prime}(T)=4 W(T)-n(n-1)$ and $\operatorname{Gut}(T)=4 W(T)-(2 n-$ $1)(n-1)$, we obtain the following corollaries.

Corollary 3.5. Let $T$ be a tree of order $n$ and diameter $d$. Then the degree distance $D^{\prime}(T)$ is at most:

(i) $4 n^{2}-11 n+12$ if $d=3$;

(ii) $7 n^{2}-8 n \sqrt{n-1}-11 n+8 \sqrt{n-1}+4$ if $d=4$;

(iii) $8 n^{2}-8 n^{3 / 2}+O(n)$ if $d=5$;

(iv) $11 n^{2}-8 \sqrt{6} n^{3 / 2}-7 n+O\left(n^{1 / 2}\right)$ if $d=6$.

Corollary 3.6. Let $T$ be a tree of order $n$ and diameter $d$. Then the Gutman index $\operatorname{Gut}(T)$ is at most:

(i) $3 n^{2}-9 n+11$ if $d=3$;

(ii) $6 n^{2}-8 n \sqrt{n-1}-9 n+8 \sqrt{n-1}+3$ if $d=4$;

(iii) $7 n^{2}-8 n^{3 / 2}+O(n)$ if $d=5$;

(iv) $10 n^{2}-8 \sqrt{6} n^{3 / 2}-5 n+O\left(n^{1 / 2}\right)$ if $d=6$. 


\section{References}

[1] V. Andova, D. Dimitrov, J. Fink and R. Skrekovski, 'Bounds on Gutman index', MATCH Commun. Math. Comput. Chem. 67 (2012), 515-524.

[2] O. Bucicovschi and S. M. Cioabă, 'The minimum degree distance of graphs of given order and size', Discrete Appl. Math. 156 (2008), 3518-3521.

[3] P. Dankelmann, I. Gutman, S. Mukwembi and H. C. Swart, 'On the degree distance of a graph', Discrete Appl. Math. 157 (2009), 2773-2777.

[4] P. Dankelmann, I. Gutman, S. Mukwembi and H. C. Swart, 'The edge-Wiener index of a graph', Discrete Math. 309 (2009), 3452-3457.

[5] E. DeLaViña and B. Waller, 'Spanning trees with many leaves and average distance', Electron. J. Combin. 15 (2008), 1-16.

[6] I. Gutman, 'Selected properties of the Schultz molecular topological index', J. Chem. Inf. Comput. Sci. 34 (1994), 1087-1089.

[7] D. J. Klein, Z. Mihalić, D. Plavšić and N. Trimajstrić, 'Molecular topological index, a relation with the Wiener index', J. Chem. Inf. Comput. Sci. 32 (1992), 304-305.

[8] M. J. Morgan, S. Mukwembi and H. C. Swart, 'On the eccentric connectivity index of a graph', Discrete Math. 311 (2011), 1229-1234.

[9] S. Mukwembi, 'On the upper bound of Gutman index of graphs', MATCH Commun. Math. Comput. Chem. 68 (2012), 343-348.

[10] J. Plesník, 'Critical graph of given diameter', Acta Math. Univ. Comenian. (N.S.) 30 (1975), 7193.

[11] I. Tomescu, 'Some extremal properties of the degree distance of a graph', Discrete Appl. Math. 98 (1999), 159-163.

[12] T. Zhou, J. Xu and J. Liu, 'On diameter and average distance of graphs', OR Trans. 8 (2004), 1-6.

\section{SIMON MUKWEMBI, School of Mathematics, Statistics and Computer Science, University of KwaZulu-Natal, Durban, South Africa \\ e-mail: Mukwembi@ukzn.ac.za}

TOMÁŠ VETRÍK, Department of Mathematics and Applied Mathematics, University of Pretoria, Pretoria, South Africa

e-mail: tomas.vetrik@gmail.com 